$\begin{gathered}\text { Revista do Departamento de Geografia } \\ \text { Universidade de São Paulo } \\ \text { www.revistas.usp.br/rdg } \\ \text { ISSN 2236-2878 }\end{gathered}$
Volume Especial - XVII SBGFA / I CNGF (2017)

\title{
Hidrodinâmica da Confluência dos Rios Cuiabá e Paraguai, Pantanal Mato-grossense
}

\section{Confluence Hydrodynamic of the Cuiabá and Paraguay Rivers, Mato-grossense Pantanal}

\author{
Leandro Domingos Luz \\ Universidade Estadual de Maringá \\ leandroluz07@gmail.com \\ José Cândido Stevaux \\ Universidade Estadual Paulista "Júlio de Mesquita Filho" Rio Claro \\ jcstevaux@gmail.com \\ Mauro Parolin \\ Universidade Estadual do Paraná - Campo Mourão \\ mauroparolin@gmail.com \\ Aguinaldo Silva \\ Universidade Federal do Mato Grosso do Sul - Campus Pantanal \\ aguinald_silva@yahoo.com.br
}

Recebido (Received): 18/03/2017

Aceito (Accepted): 01/06/2017

DOI: $10.11606 /$ rdg.v0ispe.132949

Resumo: Zonas de confluência são marcadas pela forte interação de matéria e energia de canais com características distintas e se apresentam como ambiente único no contexto da bacia de drenagem. No caso do Pantanal, a incidência sazonal dos pulsos de inundação contribui para maior dinâmica entre os fluxos e até então sem estudos com esse espectro. Nesse sentido, foram executadas duas campanhas, na vazante (novembro 2015) e outra na cheia (abril de 2016), com a finalidade de caracterizar a hidrodinâmica da zona de confluência com uso da sonda Acoustic Doppler for Current Profiler. Os dados primários foram usados nas equações para caracterizar as condições hidráulicas e de geometria de canal. Observou-se que o rio Cuiabá, embora seja tributário do Paraguai, possui maior dominância no fluxo durante a vazante, bem como maior vazão e velocidade. A dinâmica entre os fluxos dos dois canais tem contribuído com a inundação perene na porção norte do Pantanal.

Palavras-chave: ADCP; Pulsos de Inundação; Hidrodinâmica de Zonas de Confluência; Rios Meandrantes.
Abstract: Confluence zones are marked by the strong interaction of matter and energy of channels with distinct characteristics and are presented as a unique environment in the context of the drainage basin. In the case of the Pantanal wetlands, the incidence of seasonal flood pulses contributes to the hydrodynamics of the streams and until now there is no studies with that spectrum in the area. In that sense, two campaigns were carried out to the study area, one in the drier season (November 2015) and another one in the wetter season (April 2016), with the purpose to characterize the hydrodynamics of the confluence zone using the Acoustic Doppler for Current Profiler sounder. The primary data were used in the equations to characterize the hydraulic and channel geometry conditions. It was observed the Cuiabá river, although being a tributary channel of Paraguay, has greater dominance in the flow during the drier season, as well as greater flow and velocity. The dynamics between the flows of the two channels has contributed to the perennial flood in the northern portion of the Pantanal.

Keywords: ADCP; Flood Pulses; Hydrodynamics of Confluence Zones; Meandering Rivers. 


\section{INTRODUÇÃO}

As confluências são importantes pontos de interação entre matéria e energia entre fluxos distintos (BEST, 1987) e estão sujeitas a rápidas mudanças na estrutura de fluxo, transporte de sedimentos e morfodinâmica (NAZARI-GIGLOU et al., 2016). Nas últimas décadas houve maior interesse dos geomorfólogos, engenheiros fluviais e ecólogos no melhor entendimento da complexa estrutura de fluxo e geomorfologia das zonas de confluências (RICE et al., 2008).

A estrutura de fluxo na zona hidrodinâmica de confluência é influenciada por diversos fatores morfológicos (RHOADS e SUKHODOLOV, 2001). Tais morfologias incluem: a) o estilo da plataforma de confluência, que pode ser simétrico (Y) ou assimétrico (y) (MOSLEY, 1976); b) o ângulo de junção (BEST, 1987); c) a razão de fluxo nas confluências (BEST, 1987) e o d) grau de concordância do leito na entrada da confluência, que pode ser concordante, mesma elevação, ou discordante, diferentes elevações (BIRON et al., 1996).

Internacionalmente, o estudo das confluências tem ganhado destaque desde a década de 1970 (MOSLEY, 1976). No entanto, no Brasil, os estudos sobre confluências estão limitados a bacia do Alto Paraná (TURRA et al. 1999; BARROS, 2006; FRANCO, 2007; PAES, 2007; STEVAUX et al., 2009a, 2009b; SANTOS, 2015; MORAIS et al., 2016) e a bacia Amazônia (SOARES, 2007; BOUCHEZ et al., 2010; FRANZINELLI, 2011; FERREIRA, 2013). Este estudo visa contribuir para preencher a lacuna referente aos estudos de confluência no Pantanal, a partir da caracterização da estrutura de fluxo da confluência dos rios Cuiabá e Paraguai objetiva-se compreender a hidrodinâmica da zona de confluência e as consequências hidrológicas para o Pantanal.

\section{ÁREA DE ESTUDOS}

A área estudada está localizada na divisa entre os estados de Mato Grosso e Mato Grosso do Sul, entre as coordenadas $17^{\circ} 06^{\prime}$ e $18^{\circ} 08^{\prime}$ de latitude Sul e $57^{\circ} 06^{\prime}$ e $57^{\circ} 23^{\prime}$ de longitude Oeste, próximo a Serra do Amolar e a fronteira com a Bolívia (Figura 1).

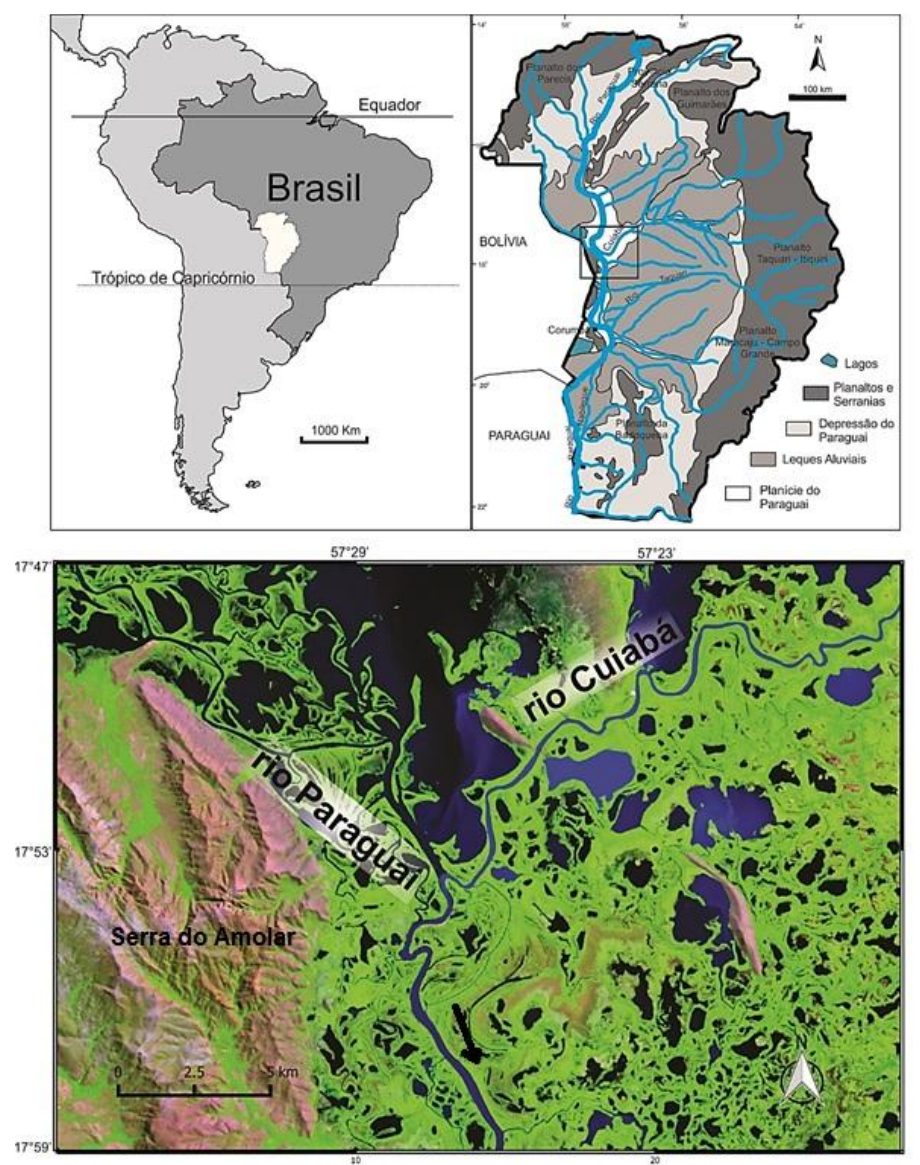

Figura 1: Mapa de localização da área de estudo. Imagem OLI/Landsat 8 em cores compostas $R(6) \mathrm{G}(5) \mathrm{B}(4)$ do dia 15/09/2015). 
O rio Cuiabá drena uma área de aproximadamente $36.000 \mathrm{~km}^{2}$ nasce a altitude aproximada de 500 m.s.n.m. na Província Serrana (PUPIM, 2014). Em sua porção superior se comporta como típico rio de planalto, percorrendo vasta superfície aplainada, denominada de Depressão Cuiabana, quando adentra a Bacia do Pantanal torna-se rio típico de planície com padrão meandrante construindo um sistema deposicional no formato de megaleque fluvial (ASSINE, 2003).

De acordo com classificação geomorfológica do megaleque do rio Cuiabá proposta Pupim (2014), a área de estudo está localizada na porção distal do sistema deposicional, compondo o lobo atual do megaleque, com formação de planície desconfinada. O clima regional da área de estudo pode ser classificado como Aw (Tropical sazonal - na classificação de Köppen-Geiger), com estação seca entre os meses de maio a setembro, com elevadas precipitações durante os meses de novembro, dezembro e janeiro. A precipitação média anual é de $1.250 \mathrm{~mm}$ com temperaturas médias entre $21,4^{\circ} \mathrm{C}$ (julho) e $27,4^{\circ} \mathrm{C}$ (dezembro) (JUNK E CUNHA, 2005).

\section{MATERIAIS E MÉTODOS}

Foram executadas duas campanhas a área de estudo: uma em novembro de 2015, no período de vazante, e outra em abril de 2016 no período de cheia. Na primeira campanha foram estabelecidos 5 transectos na zona de confluência, dois em cada canal e um após a confluência. Já na segunda campanha foram acrescidos de dois transectos após a confluência para a melhor descrição da junção de fluxos (Figura 2). Nas seções transversais foi utilizado sonda ADCP (Acoustic Doppler for Current Profile) modelo Rio Grande ${ }^{\circledR} 600 \mathrm{Kz}$, acoplado a um computador portátil com o uso do software WinRiver II® v 2.17 e GPS portátil modelo Garmim ${ }^{\circledR}$ 60cs. Em novembro de 2015 foram monitoradas duas seções no rio Cuiabá, 11 e 12A, uma anterior a confluência no Rio Paraguai (seção 13) e duas no rio Paraguai a jusante da confluência (12B e 14). No entanto, com vista a melhor caracterização da junção dos fluxos e comportamento hidrossedimentar foram acrescidas duas seções, 15 e 16, no período da cheia em abril de 2016. A metodologia de coleta dos dados com ADCP foi com embarcação em movimento (MUELLER et al., 2013), que é amplamente utilizada em grandes rios. A bússola interna do aparelho foi trocada pela referência dada pelo GPS externo. A partir dos dados do ADCP foram calculados os parâmetros hidráulicos de: potência de canal; potência de canal específica e parâmetros de comportamento de fluxo (Reynolds e Froude), bem como a razão de descarga e razão de momentum da confluência.

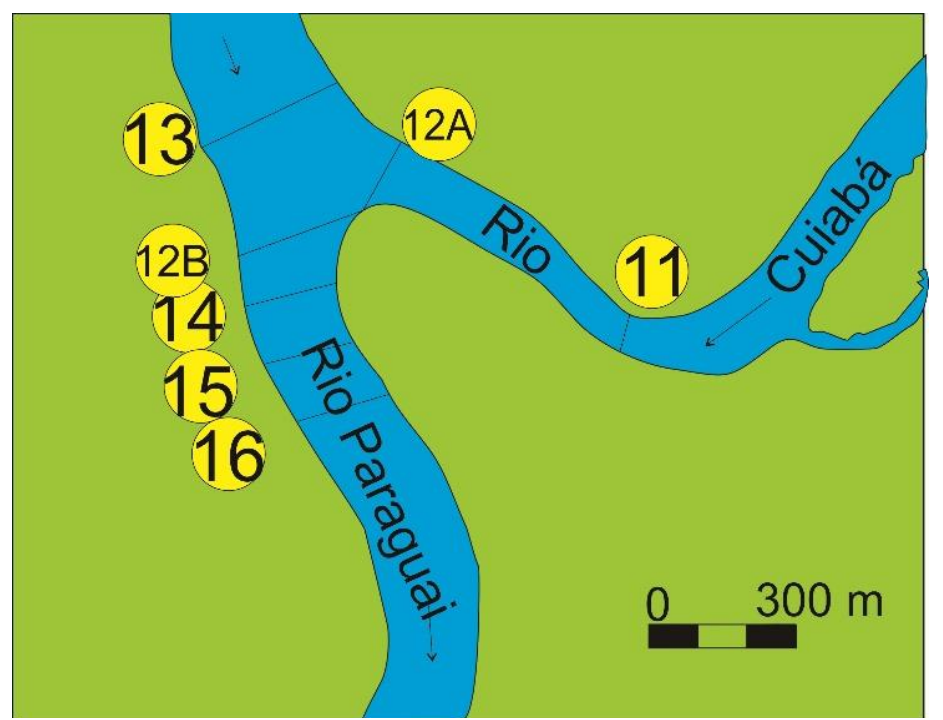

Figura 2: Posição das seções na confluência dos rios Cuiabá e Paraguai.

A partir dos dados primários obtidos com o ADCP foram aplicadas as seguintes equações: potência do canal $(\Omega)$, dada pela seguinte Equação 1: 


$$
\Omega=\rho g Q S
$$

\section{Equação 1}

Onde: $\rho$ densidade da água; g aceleração devido à gravidade; $Q$ vazão média da seção e $S$ a declividade da linha da água. Unidade de medida em W/s.

Potência de canal especifica $(\omega)$. Dada pela seguinte Equação 2:

$$
\omega=\mathbf{\Omega} / w \quad \text { Equação 2 }
$$

Onde: $\Omega$ é a potência do canal e $w$ é a largura do canal. Unidade de medida em W/m2.

A declividade da linha d'água (S) foi obtida a partir da equação de Manning (Equação 3):

$$
v=\left(R^{\wedge} 0,67 S^{\wedge} 0,5\right) / n \quad \text { Equação } 3
$$

Onde: $v$ é a velocidade do canal; $R$ é o raio hidráulico e $n$ é o coeficiente de Manning.

\section{Equação 4:}

O número de Froude (Fr) é dado pela razão entre a força inercial e gravitacional, expressa pela

$$
F r=v / \sqrt{g} d
$$

\section{Equação 4}

Onde: $v$ é a velocidade, $g$ é a constante gravitacional e $d$ é a profundidade. O número de Froude permite analisar se o fluxo é subcrítico (<1), crítico (=) ou supercrítico (>1).

O número de Reynolds $(R e)$ expressa a razão entre a força inercial e a força da viscosidade que age sobre o fluído, o qual permite analisar se o fluxo é laminar, transicional ou turbulento (CHARLTON, 2008). O número de Reynolds é obtido pela Equação 5:

$$
R e=(\text { Força inercial }) /(\text { Força viscosa }) \rightarrow R e=v R / z \quad \text { Equação } 5
$$

Onde: $v$ é a velocidade média do fluxo, $R$ é Raio hidráulico e $z$ é a viscosidade cinemática. Com número de Reynolds $<500$ o fluxo é laminar dominado pela força viscosa. Quando apresenta um valor >2100 a força inercial é maior e o fluxo se torna turbulento. A transição entre o fluxo laminar e turbulento ocorre entre 500 a 2000. Como o número de Reynolds não apresenta dimensão, portanto, não possui unidades (CHARLTON, 2007).

Best (1987) estabelece dois parâmetros hidráulicos no estudo das confluências: Razão da descarga ( $Q r)$ dada pela Equação 6:

$$
Q r=Q t / Q m
$$

Equação 6

Onde: $Q t$ é a vazão do canal tributário e $Q m$ é a vazão do canal principal (main channel). Valores de $\mathrm{Qr} \geq 1$ o canal tributário domina o fluxo.

Razão de momentum $(M r)$ é dada pela Equação 7:

$$
M r=\left(\rho_{t} Q_{t} U_{t}\right) /\left(\rho_{m} Q_{m} U_{m}\right)
$$

Onde: $\rho$ é a densidade do fluído, $Q$ descarga média e $U$ é a velocidade média. Os subscritos t e m referem-se a canal tributário e canal principal. 


\section{RESULTADOS E DISCUSSÕES}

A confluência dos rios Cuiabá e Paraguai é marcada pela forte sazonalidade dos pulsos de inundação que ocorrem na planície do Pantanal. O rio Cuiabá é o principal afluente do Paraguai na porção superior do Pantanal e a zona de confluência localiza-se em um dos dois grandes gargalhos que compõem a hidrologia do Pantanal (ver publicação de ASSINE et al., 2016). Segundo Nobre e Shukla (1996), a dinâmica dos pulsos sazonais de inundação que ocorrem no Pantanal está diretamente relacionada ao efeito do tipo monsônico causado pela incidência da Zona de Convergência Intertropical e a Zona de Convergência do Atlântico Sul. O período chuvoso se estende de outubro a março, enquanto que entre os meses de julho e setembro são os meses mais secos (ASSINE et al., 2016).

Ao analisar a série histórica das duas estações mais próximas da confluência (Figura 3), observa-se que há significativa diferença na onda de cheia dos dois canais.

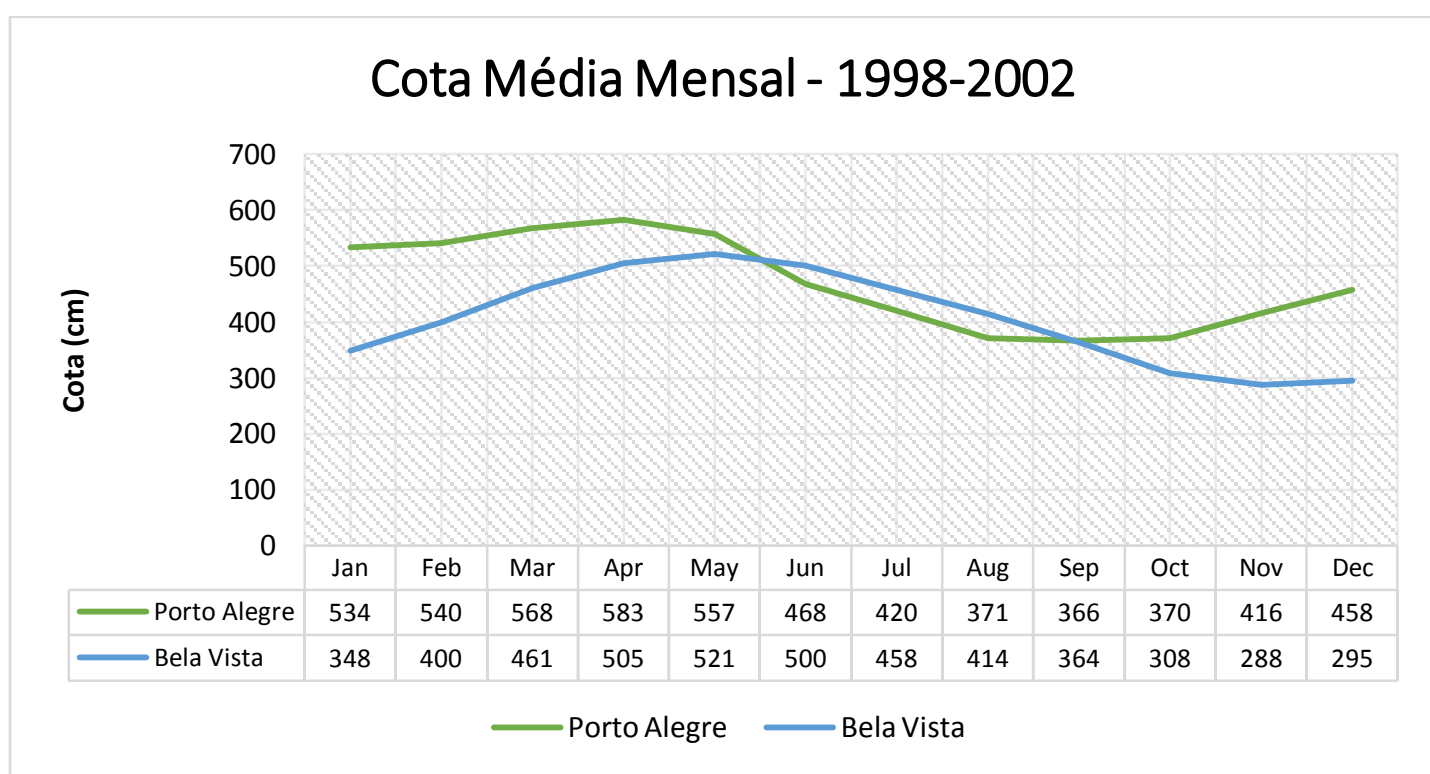

Figura 3: Hidrográfico das duas estações fluviométricas mais próximas da confluência, Porto Alegre no rio Cuiabá e Bela Vista do Norte no rio Paraguai. Agência Nacional de Águas - ANA

(http://www.snirh.gov.br/hidroweb/, recuperado em 15 de dez. 2016).

A diferença na onda de cheia permite que no período de vazante, em novembro de 2015 o rio Cuiabá apresentasse Razão de Descarga (Qr) de 1,4 o que infere ao tributário a dominância do fluxo na confluência e Razão de Momentum (Mr) com valore de 4,41. O domínio do tributário sobre o canal principal pode estar relacionado ao fato da onda de inundação chegar primeiro pelo rio Cuiabá, uma vez que o rio Paraguai percorre uma distância maior até a confluência e no percurso adentra da lagoa Gaíva em ambiente flúviolacustre. Por sua vez, o Paraguai domina o fluxo no período de cheia, na medição de abril de 2016 apresentou valor de 0,75 para a razão de descarga e 0,5 para a razão de momentum.

As duas seções analisadas no rio Cuiabá (11 e 12A) apresentaram valores maiores de velocidade de fluxo na seca $(0,59 \mathrm{~m} / \mathrm{s}$ e $0,71 \mathrm{~m} / \mathrm{s}$, respectivamente). Enquanto que na cheia os valores maiores foram encontrados nas seções no rio Paraguai (12B, 13, 14, 15, 16) (Tabela 1). 
Tabela 1: Razão largura/profundidade, vazão média e velocidade média.

\begin{tabular}{|c|c|c|c|c|c|c|c|}
\hline \multirow{2}{*}{ Seção } & \multirow{2}{*}{$\begin{array}{c}\text { Largura } \\
(\mathbf{m})\end{array}$} & \multirow{2}{*}{$\begin{array}{c}\text { Prof. Média } \\
(\mathbf{m})\end{array}$} & \multirow{2}{*}{$\begin{array}{c}\text { Largura / } \\
\text { Profundidade }\end{array}$} & \multicolumn{2}{|c|}{ Vazão $\left(\mathbf{m}^{\mathbf{3}} / \mathbf{s}\right)$} & \multicolumn{2}{|c|}{$\begin{array}{c}\text { Velocidade média } \\
(\mathbf{m} / \mathbf{s})\end{array}$} \\
\cline { 5 - 8 } & & & & Nov/15 & Abr/16 & Nov/15 & Abr/16 \\
\hline 11 & 95,1 & 7,67 & 12,40 & 434,0 & 467,2 & 0,59 & 0,51 \\
\hline 12A & 155,4 & 5,66 & 27,45 & 478,0 & 396,5 & 0,71 & 0,54 \\
\hline 12B & 346,3 & 5,95 & 58,20 & 852,0 & $1.384,0$ & 0,50 & 0,70 \\
\hline 13 & 329,9 & 8,15 & 40,47 & 341,8 & 926,6 & 0,17 & 0,34 \\
\hline 14 & 191,0 & 9,41 & 20,29 & 832,5 & $1.368,3$ & 0,48 & 0,77 \\
\hline 15 & 204,0 & 8,65 & 23,58 & - & $1.450,1$ & - & 0,85 \\
\hline 16 & 200,7 & 9,55 & 20,51 & - & $1.464,5$ & - & 0,88 \\
\hline
\end{tabular}

De acordo com a descrição das zonas hidrodinâmicas em confluências proposta por Rhoads e Sukhodolov (2001), a zona de separação de fluxo a jusante da confluência é bem marcada na seção 14 durante o período de vazante, com velocidades mais baixas. Nas seções $12 \mathrm{~B}$ e 14 que são perpendiculares à zona de escavação, a velocidade é menor na vazante, $0,50 \mathrm{~m} / \mathrm{s}$ e $0,48 \mathrm{~m} / \mathrm{s}$, respectivamente e maiores na cheia $0,70 \mathrm{~m} / \mathrm{s}$ e $0,77 \mathrm{~m} / \mathrm{s}$. Na zona de restabelecimento de fluxo, após a confluência as velocidades voltam a aumentar. Ao observar a porção média da seção 12-12' nas Figuras 4 e 5 é possível verificar a sazonalidade na força exercida sobre a junção dos dois fluxos. Na vazante (nov.15) o rio Cuiabá apresenta maiores valores de velocidade média. Já na cheia (abr. 16) o fluxo do rio Cuiabá se concentra próximo a porção média da seção 12-12'. Isso se deve pela maior vazão do rio Paraguai que passa a ter maior razão de descarga que seu tributário durante o período de cheia.

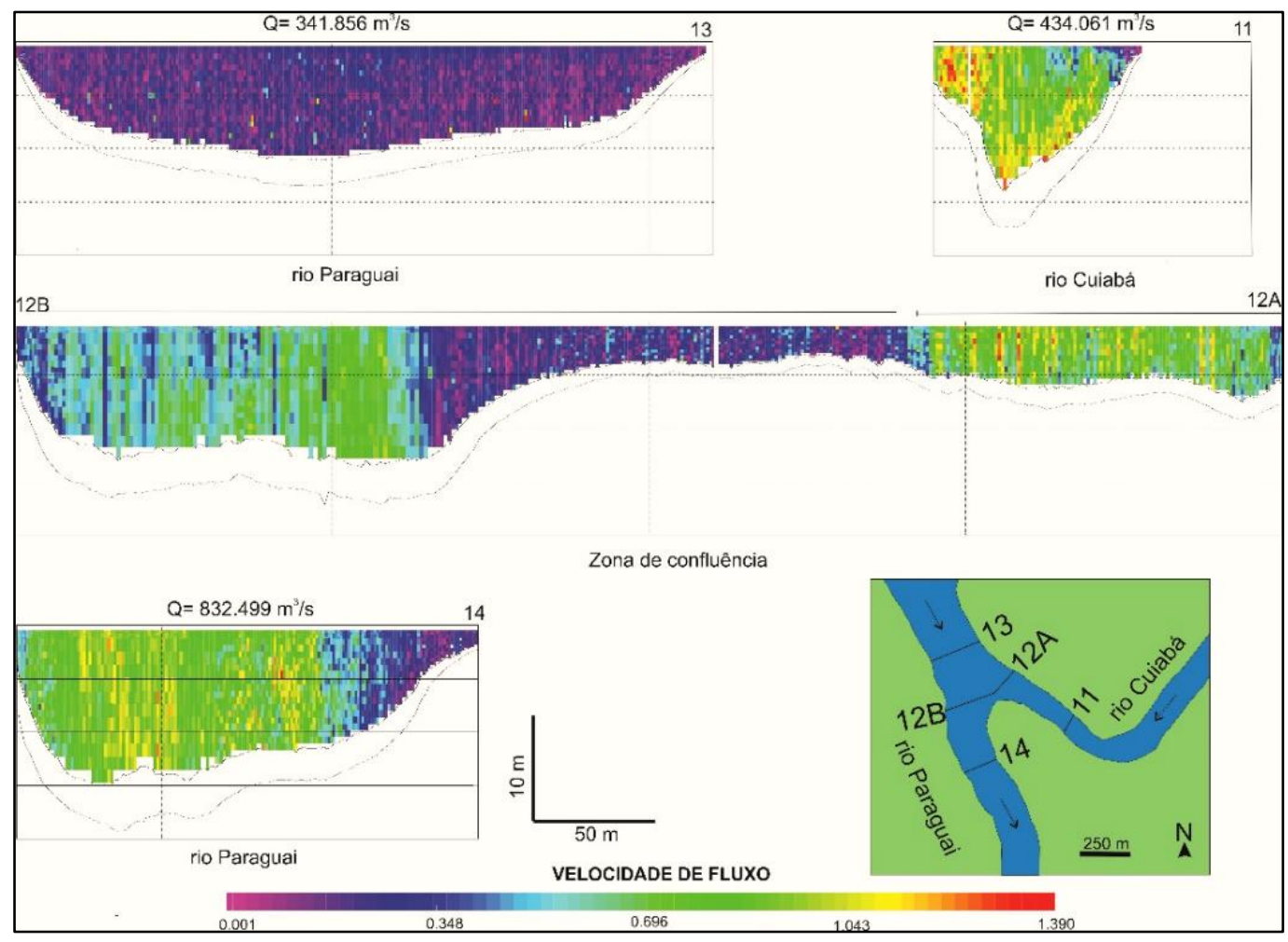

Figura 4: Estrutura do fluxo das seções analisadas na confluência dos rios Cuiabá e Paraguai em novembro de 2015. 


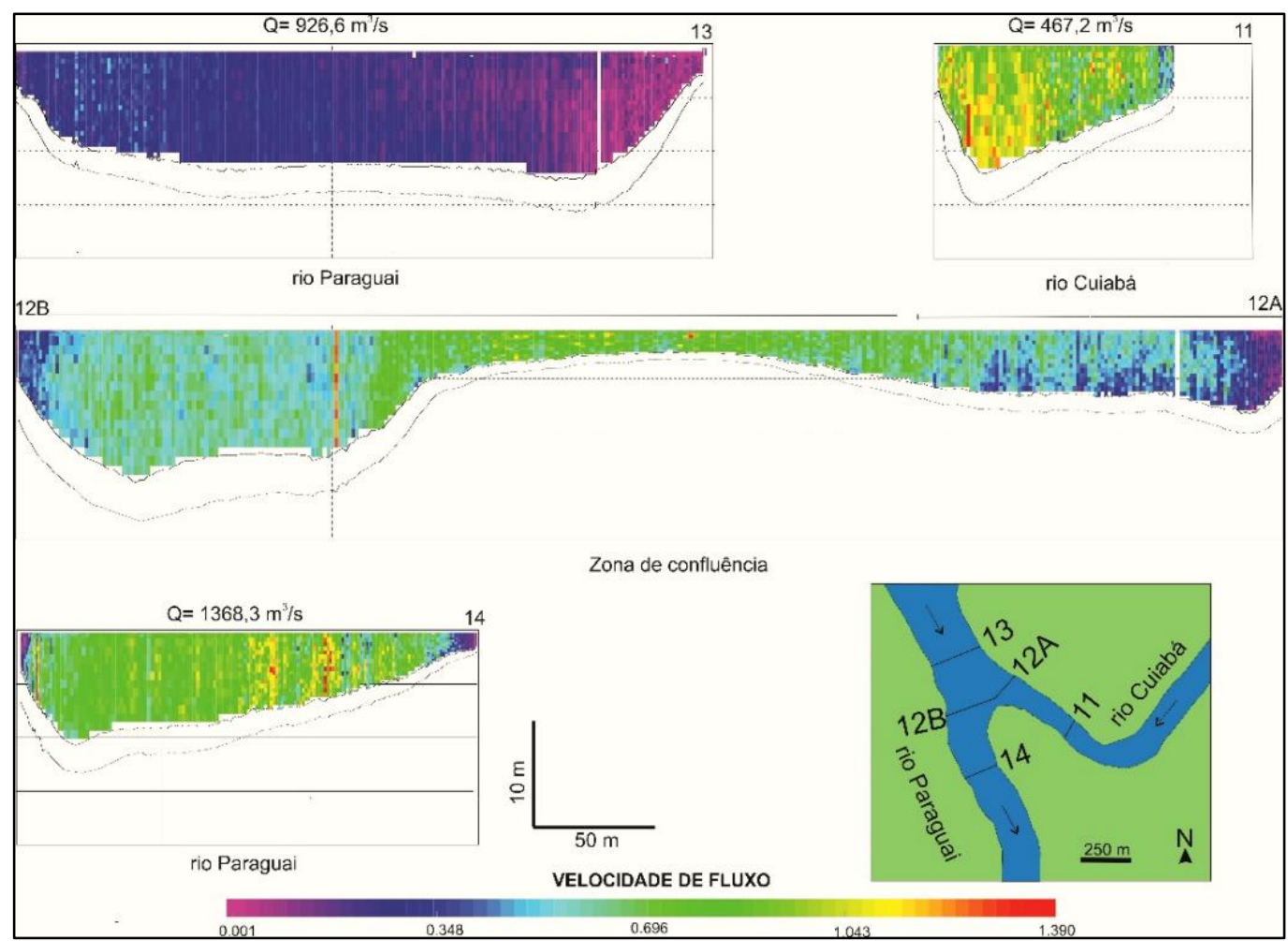

Figura 5: Estrutura do fluxo das seções analisadas na confluência dos rios Cuiabá e Paraguai em abril de 2016.

Ao analisar a direção de fluxo na confluência, nota-se que durante o período de vazante o rio Cuiabá domina a direção de fluxo (Figura 6). Já no período de cheia, as direções de fluxo do rio Cuiabá são diretamente influenciadas pelo rio Paraguai, o qual apresenta valores superiores de velocidade de fluxo.
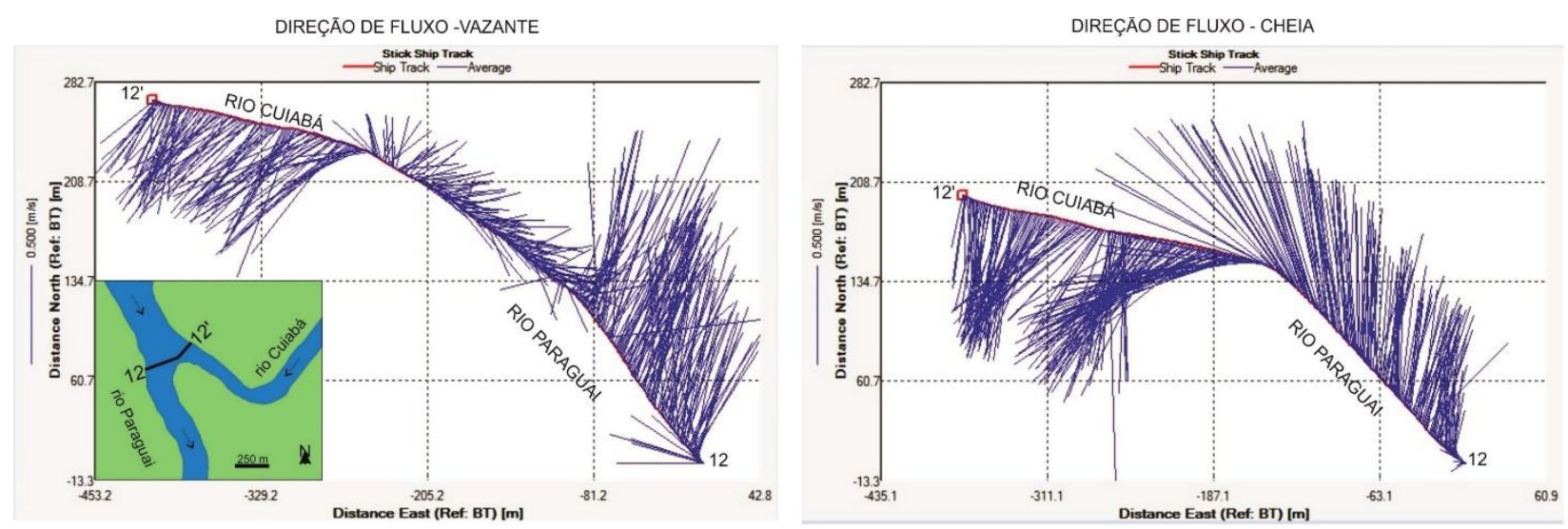

Figura 6: Direção de fluxo na confluência no período de vazante e cheia.

Em relação aos parâmetros hidráulicos, a seção 13 no rio Paraguai, anterior a confluência, apresentou menor potência de canal específica (entre $0,01 \mathrm{~W} / \mathrm{m}^{2}$ e $0,11 \mathrm{~W} / \mathrm{m}^{2}$ ) (Tabela 2). A maior potência de canal específica foi registrada na seção 16 após a confluência que, segundo Best $(1987,1988)$, compõem a zona de aceleração de fluxo. 
Tabela 2: Parâmetros hidráulicos das seções.

\begin{tabular}{|c|c|c|c|c|c|c|}
\hline \multirow{2}{*}{ Seção } & \multicolumn{2}{|c|}{ Declividade (m/m) } & \multicolumn{2}{c|}{ Potência de Canal (W/s) } & \multicolumn{2}{c|}{$\begin{array}{c}\text { Potência de Canal Específico } \\
\left(\mathbf{W} / \mathbf{m}^{2}\right)\end{array}$} \\
\cline { 2 - 7 } & Nov/15 & Abr/16 & Nov/15 & Abr/16 & Nov/15 & Abr/16 \\
\hline 11 & 0,000012 & 0,000008 & 48,26 & 32,4 & 0,54 & 0,35 \\
\hline 12A & 0,000036 & 0,000012 & 208,6 & 68,0 & 1,35 & 0,42 \\
\hline 12B & 0,000013 & 0,000018 & 165,2 & 289,1 & 0,47 & 1,06 \\
\hline 13 & 0,000001 & 0,000004 & 4,6 & 36,5 & 0,01 & 0,11 \\
\hline 14 & 0,000010 & 0,000020 & 79,3 & 281,2 & 0,41 & 1,37 \\
\hline 15 & - & 0,000027 & - & 381,9 & - & 1,90 \\
\hline 16 & - & 0,000027 & - & 395,4 & - & 1,95 \\
\hline
\end{tabular}

Ao analisar a planície do rio Paraguai entre a Baia Vermelha e a foz do rio Miranda, Macedo (2013) assevera que neste ponto o rio Paraguai apresenta baixa potência de canal, o que confere a capacidade de transportar apenas areia de granulometria fina. Esses dados estão em consonância com os resultados encontrados na área de estudo. Em contrapartida, no médio Cuiabá, quando o canal passa de meandrante para anabranching em decorrência de uma anomalia tectônica, Meira (2015) encontrou valores aproximadamente seis vezes maiores de potência de canal, que aqueles encontrados nesse estudo.

O comportamento de fluxo pelo número de Reynolds pode ser considerado turbulento e o número de Froude indicou fluxo turbulento tranquilo (Tabela 3). Bryan e Kuhn (2002) acrescentam que quanto mais assimétrica a junção dos canais, mais complexo se torna a estrutura da zona de escavação, devido a conturbada hidrodinâmica de competição de fluxos. Os resultados sobre o comportamento de fluxo são semelhantes aos encontrados por Meira (2015) e por Macedo (2013). O que pode ser uma característica regional.

Tabela 3: Comportamento de fluxo na confluência.

\begin{tabular}{|c|c|c|c|c|}
\hline \multirow{2}{*}{ Seção } & \multicolumn{2}{|c|}{ Reynolds } & \multicolumn{2}{c|}{ Froude } \\
\cline { 2 - 5 } & Nov/15 & Abr/16 & Nov/15 & Abr/16 \\
\hline 11 & $5,31 \times 10^{9}$ & $5,31 \times 10^{9}$ & 0,068 & 0,054 \\
\hline 12A & $4,45 \times 10^{9}$ & $4,19 \times 10^{9}$ & 0,096 & 0,061 \\
\hline 12B & $4,50 \times 10^{9}$ & $7,30 \times 10^{9}$ & 0,059 & 0,074 \\
\hline 13 & $1,57 \times 10^{9}$ & $3,59 \times 10^{9}$ & 0,020 & 0,038 \\
\hline 14 & $4,98 \times 10^{9}$ & $8,40 \times 10^{9}$ & 0,052 & 0,084 \\
\hline 15 & - & $8,56 \times 10^{9}$ & - & 0,024 \\
\hline 16 & - & $9,35 \times 10^{9}$ & - & 0,024 \\
\hline
\end{tabular}

Uma das hipóteses previamente levantadas era que o rio Cuiabá possuía capacidade de descarga suficiente para dominar o fluxo na confluência. Isso foi comprovado pela razão quase duas vezes maior no período de vazante, dado a diferença no ciclo hidrográfico apresentado pelos dois rios. Nesse sentido, o gargalo que se forma nessa área é resultado da união de fatores hidrodinâmicos e geomorfológicos.

Mosley (1976) apresentou uma série de experimentos em laboratório sobre as zonas de escavação e concluiu que a profundidade tem razão direta com o ângulo de junção dos canais, no entanto se estabiliza a partir de $100^{\circ}$. No caso dos rios Cuiabá e Paraguai que possuem uma junção com angulação de aproximadamente $160^{\circ}$ essa morfologia possui uma importância singular na hidrodinâmica da confluência, que precisa ser melhor estudada. 


\section{CONCLUSÕES}

A partir dos resultados podem ser levantadas as seguintes conclusões sobre a hidrodinâmica na confluência dos rios Cuiabá e Paraguai: a diferença entre as ondas de cheia dos dois rios contribui para a hidrodinâmica da confluência; o rio Cuiabá recebe a onda de cheia antes do rio Paraguai o que contribui para que o fluxo do canal tributário seja dominante em certo período do ano; não há alteração significativa na vazão média do rio Cuiabá, na sua foz, na cheia ou na vazante; na cheia a vazão do rio Paraguai é aproximadamente 3 vezes maior que na vazante; a estrutura de fluxo é fortemente influenciada pela morfologia da confluência; a potência de canal específica do rio Cuiabá é maior na vazante e é o inverso para o rio Paraguai, o qual apresenta maior potência de canal específica na cheia; e o fluxo de ambos os canais é turbulento. A hidrodinâmica na confluência e o sistema de gargalo, próximo à área de estudo, influencia a inundação perene de grande parte da porção norte do Pantanal, bem como, na hidrologia do sistema pantaneiro, como no atraso da onda de cheia.

\section{BIBLIOGRAFIA}

ASSINE, M. L. Sedimentação na Bacia do Pantanal Mato-grossense, Centro-Oeste do Brasil. Tese LivreDocente. Departamento de Geologia Aplicada - Instituto de Geociências e Ciências Exatas - Universidade Estadual Paulista, Rio Claro, 105 p., 2003.

ASSINE, M. L.; MACEDO, H. A.; STEVAUX, J. C.; BERGIER, I.; PADOVANI, C. R.; SILVA, A. Avulsive rivers in the hydrology of the Pantanal wetland. In: BERGIER, I.; ASSINE, M.L. (Org.). Dynamics of the Pantanal wetland in South America. New York: Springer, p. 83-110. 2016.

BARROS, C. S. Dinâmica sedimentar e hidrológica na confluência do rio Ivaí com o rio Paraná, município de Icaraíma- PR. Dissertação (Mestrado em Geografia) - Programa de Pós-graduação em Geografia, Universidade Estadual de Maringá, Maringá. 69p. 2006.

BEST, J. L. Flow dynamics at river channel confluences: Implications for sediment transport and bed morphology, in recent developments in fluvial. Sedimentology, Amsterdam, v. 39, p. 27-35. 1987.

BEST, J. L.; RHOADS, B. L. Sediment transport, bed morphology and the sedimentology of river channel confluences. In: RICE, S. P.; ROY, A G.; RHOADS, B. L. (Eds) River confluences, tributaries and the fluvial network. London: John Wiley \& Sons Ltd, p. 45-72. 2008.

BEST, J. Sediment transport and bed morphology at river channel confluences. Sedimentology, Wiley Online Library, v. 35, p. 481-498. 1988.

BIRON, P; ROY, A. G.; BEST, J. L. Turbulent flow structure at concordant and discordant open-channel confluences. Experiments in Fluids, Amsterdam, v. 21, p. 437-446. 1996.

BOUCHEZ, J.; LAJEUNESSE, E.; GAILLARDET, J.; FRANCE-LANORD, C.; DUTRA-MAIA, P.; MAURICE, L. Turbulent mixing in the Amazon River: The isotopic memory of confluences. Earth and Planetary Science Letters, v. 290, n. 1, p. 37-43. 2010.

BRYAN, R. B.; KUHN, N. J. Hydraulic conditions in experimental rill confluences and scour in erodible soils. Water Resources Research, Wiley Online Library, v. 38, p. 1-13. 2002.

CHARLTON, R. Fundamentals of fluvial geomorphology. New York: Routledge, 234p. 2007.

FERREIRA, M. P. Geocronologia e proveniência dos sedimentos holocênicos da confluência dos rios Negro e Solimões, AM. Dissertação (Mestrado em Geoquímica e Geotectônica) - Instituto de Geociências, Universidade de São Paulo, São Paulo. 95p. 2013.

FRANCO, A. L. A. Análise da dinâmica e estrutura de fluxo e da morfologia da confluência dos rios Ivaí e Paraná, PR/MS. Dissertação (Mestrado em Análise Geoambiental), Universidade de Guarulhos, Guarulhos. 98p. 2007.

FRANZINELLI, E. Características morfológicas da confluência dos rios Negro e Solimões (Amazonas, Brasil). Revista Brasileira de Geociências, v. 41, n. 4, p. 587-596. 2011.

JUNK, W. J.; CUNHA, C. N. D. Pantanal: a large South American wetland at a crossroads. Ecological Engineering, Amsterdam, v. 24, n. 4, p. 391-401. 2005. 
MACEDO, H. A. Geomorfologia e hidrossedimentologia da planície do rio Paraguai, da lagoa Vermelha à confluência do rio Miranda, Pantanal Mato-grossense. Dissertação (Mestrado em Geociências e Meio Ambiente). Instituto de Geociências e Ciências Exatas, Universidade Estadual Paulista, Rio Claro. 73p. 2013.

MEIRA, F. C. Evolução geomorfológica da planície do rio Cuiabá, Pantanal Mato-grossense. Tese (Doutorado em Geociências e Meio Ambiente) - Instituto de Geociências e Ciências Exatas, Universidade Estadual Paulista, Rio Claro. 154 p. 2015.

MORAIS, E. S. de; SANTOS, M.L. dos; CREMON, É.H.; STEVAUX, J.C. Floodplain evolution in a confluence zone: Paraná and Ivaí rivers, Brazil. Geomorphology, Amsterdam, v. 257, p. 1-9. 2016.

MOSLEY, M. P. An experimental study of channel confluences. The Journal of Geology. Chicago, v. 84, p. 535-562. 1976.

MUELLER, D.; WAGNER, C. R.; REHMEL, M. S.; OBERG, K. A.; RAINVILLE, F. Measuring discharge with acoustic Doppler current profilers from a moving boat. Washington, D.C.: U.S. Department of Interior, 95 p. 2013.

NAZARI-GIGLOU, A.; JABBARI-SAHEBARI, A.; SHAKIBAEINIA, A.; BORGHEI, S. M. An experimental study of sediment transport in channel confluences. International Journal of Sediment Research, Amsterdan, v. 31, p. 87-96. 2016.

NOBRE, P.; SHUKLA, J. Variations of sea surface temperature, wind stress, and rainfall over the tropical Atlantic and South America. Journal of Climate, Boston, v. 9, p. 2464-2479. 1996.

PAES, R. J. Dinâmica sedimentar e hidrológica na confluência do rio Ivaí com o rio Paraná, município de Icaraíma- PR. Dissertação (Mestrado em Análise Geoambiental), Universidade de Guarulhos, Guarulhos. 53p. 2007.

PUPIM, F. do N. Geomorfologia e paleo-hidrologia dos megaleques dos rios Cuiabá e São Lourenço, Quaternário da bacia do Pantanal. Tese (Doutorado em Geociências e Meio Ambiente) Instituto de Geociências e Ciências Exatas, Universidade Estadual Paulista, Rio Claro. 119p. 2014.

RHOADS, B. L.; SUKHODOLOV, A.N. Field investigation of three- dimensional flow structure at stream confluences. Water Resources Research, Wiley Online Library, v. 37, n. 9, p. 2393-2410. 2001.

RICE, S.; ROY, A.; RHOADS, B. (Eds.) River confluences, tributaries and fluvial network. Sussex: John Wiley \& Sons, 456 p. 2008.

SANTOS, V. C. dos. Ambientes de confluência no contexto da rede de drenagem: exemplo da bacia hidrográfico do rio Ivaí - estado do Paraná. Tese (doutorado em Geociências e Meio Ambiente). Instituto de Geociências e Ciências Exatas, Universidade Estadual Paulista, Rio Claro. 458 p. 2015.

SOARES, E. A. A. Depósitos pleistocenos da região de confluência dos rios Negro e Solimões, Amazonas. Tese (Doutorado em Geologia Sedimentar) - Instituto de Geociências, Universidade de São Paulo, São Paulo. 205p. 2007.

STEVAUX, J. C.; AMÂNCIO, A.; ETCHEBEHERE, M. L. C.; FUJTTA, R. H. Flow structure and dynamics in large tropical river confluence: example of the Ivaí and Paraná rivers, Southern Brazil. Geociências, São Paulo, v. 28, p. 1-13. 2009a.

STEVAuX, J. C.; PAES, R. J.; FRANCO, A. A.; ETCHEBEHERE, M. L. C.; FUJITA, R. H. Morphodynamics in the Confluence of large regulated Rivers: the case of Parana and Paranapanema Rivers. Latin American Journal of Sedimentology and Basin Analysis, v. 16, n. 2, p. 101-109. 2009 b.

TURRA, T. M.; MARQUES, V. V.; STEVAUX, J. C. Confluence bar of the São Pedro Brook in the Paraná River: Genesis and environmental importance. Boletim Goiano de Geografia, v. 19, n. 1, p. 50-54. 1999. 